\title{
Erratum: Chiral spin liquids at finite temperature in a three-dimensional Kitaev model [Phys. Rev. B 96, 174409 (2017)]
}

\author{
Yasuyuki Kato, Yoshitomo Kamiya, Joji Nasu, and Yukitoshi Motome
}

(Received 29 March 2018; published 12 April 2018)

DOI: 10.1103/PhysRevB.97.139902

In our paper, there is a missing interaction in the low-energy effective model for the large $J_{z}$ limit. Accordingly, the effective model [Eqs. (3) and (5) in the original paper], some sentences in Sec. III A, and Fig. 3 need to be corrected. Although the ground-state flux configuration for the large $J_{z}$ limit is changed by the correction, this error does not affect our main conclusion: The effective model has no frustration, and the system undergoes a strong first-order phase transition from the paramagnetic phase to the chiral spin liquid (CSL).

The low-energy effective model for the large $J_{z}$ limit is corrected by adding a missing four-body interaction: Eqs. (3) and (5) in the original paper are replaced by

$$
\begin{aligned}
\mathcal{H}_{\mathrm{eff}}^{z}= & J \sum_{\left\langle p, p^{\prime}\right\rangle} b_{p} b_{p^{\prime}}-J^{\prime} \sum_{\left(p, p^{\prime}\right)} b_{p} b_{p^{\prime}} \\
& -J^{(4)} \sum_{\left\langle p_{1}, p_{2}, p_{3}, p_{4}\right\rangle} b_{p_{1}} b_{p_{2}} b_{p_{3}} b_{p_{4}},
\end{aligned}
$$

and

$$
J=\frac{33}{2048} \frac{J_{x}^{4} J_{y}^{4}}{\left|J_{z}^{7}\right|}, \quad J^{\prime}=\frac{9}{33} J, \quad J^{(4)}=\frac{1}{2} J,
$$

respectively. The sum of $\left\langle p_{1}, p_{2}, p_{3}, p_{4}\right\rangle$ runs over all the faces of $\mathrm{B}$ and $\mathrm{R}$ cubes where $p_{1}-p_{4}$ indicate the corners of each square face.

In Sec. III A 1, the text after the second sentence, "The model has...," is replaced by "As $J$ and $J^{(4)}$ are relatively larger than $J^{\prime}$ in the model in Eq. (3), the lowest-energy flux configuration is a simple staggered one shown in the inset of Fig. 3. The ground-state energy per $b_{p}$ is simply computed as," and Eq. (8) is replaced by

$$
\varepsilon_{\mathrm{GS}}^{z}=-\frac{3}{2}\left(J-J^{\prime}+J^{(4)}\right)=-\frac{81}{44} J .
$$

Figure 3 in the original paper is replaced by the figure below.
Finally, in Sec. III A 2, the sentence starting from "Without the constraints, ..." is replaced by "Since a similar strong firstorder phase transition to a CSL is seen in the MC simulations with $J^{(4)}=0$, the four-body interaction is not the origin of the strong discontinuity. In addition, without the constraints and the $J^{(4)}$ term, $\mathcal{H}_{\text {eff }}^{z}$ is merely an unfrustrated Ising model, which undergoes a continuous transition."

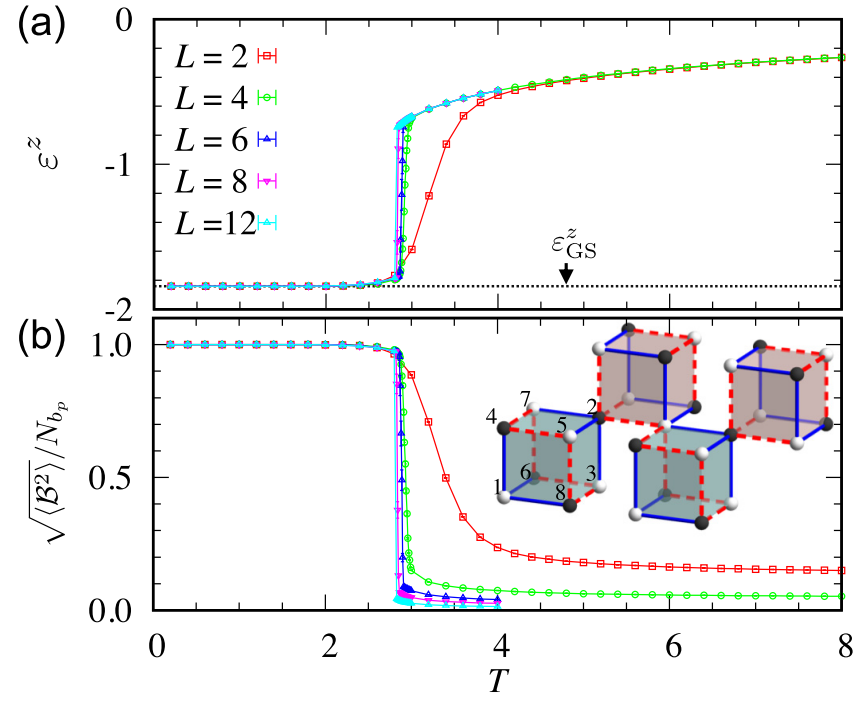

FIG. 3. Temperature dependence of (a) the energy density $\varepsilon^{z}$ and (b) the order parameter $\sqrt{\left\langle\mathcal{B}^{2}\right\rangle} / N_{b_{p}}$ for the large- $J_{z}$ effective model $\mathcal{H}_{\text {eff }}^{z}$ in Eq. (3). We set $J=1\left(J^{\prime}=9 / 33, J^{(4)}=1 / 2\right)$. $\varepsilon_{\mathrm{GS}}^{z}$ indicates the ground-state energy in Eq. (8) for the $b_{p}$ configuration in the inset of (b); the black (white) spheres represent $b_{p}=-1(+1)$ [or $\left.b_{p}=+1(-1)\right]$, and the numbers denote the sublattices. 